\title{
Identification of Etiologic Agents of the Pertussis-like Syndrome in Children by Real-time PCR Method
}

\section{Shima Mahmoudi ${ }^{1}$, Maryam Banar ${ }^{1}$, Babak Pourakbari ${ }^{1}$, Hediyeh} Sadat Alavi ${ }^{2}$, Hamid Eshaghi ${ }^{2}$, Alireza Aziz Ahari ${ }^{3}$, Setareh Mamishi ${ }^{1,2}$ ${ }^{1}$ Pediatric Infectious Disease Research Center, Tehran University of Medical Sciences, Tehran, Iran;

${ }^{2}$ Department of Infectious Diseases, Pediatrics Center of Excellence, Children's Medical Center Hospital, Tehran University of Medical Sciences, Tehran, Iran; ${ }^{3}$ Department of Radiology, Rasool-e-Akram Hospital, Iran University of Medical Sciences, Tehran, Iran

Received November 14, 2017; Accepted March 21, 2018.

Key words: Pertussis-like syndrome $-B$. pertussis - Adenovirus - Respiratory syncytial virus - Human metapneumovirus - Influenza virus A

Abstract: The aim of this study was to recognize the identity and frequency of etiologic agents of the pertussis-like syndrome in children $<2$ years of age. A cross-sectional hospital-based study conducted from August 2014 to August 2015. All children $<2$ years of age $(n=100)$ who were suspected as pertussis infected were enrolled in this study and tested for Bordetella pertussis, adenovirus (Adv), respiratory syncytial virus (RSV), human metapneumovirus (hMPV), and influenza virus $A$ (INF-A) by real-time PCR technique. RSV was the most detected pathogen (20\%), followed by B. pertussis (18\%), Adv (16\%), INF-A (11\%), and hMPV (10\%).

Co-infection was observed in 8 patients $(11 \%)$ and the combinations of RSV/INF-A $(n=3,4 \%)$, and $A d V / B$. pertussis $(n=3,4 \%)$ were more frequent. RSV, B. pertussis, and hMPV were more frequent pathogens among infants $<4$ months of age. However, Adv and INF-A were more frequent pathogens among children $>6$ months of age. In this study, RSV was the most frequent identified pathogen $(n=20,20 \%)$, followed by $B$. pertussis $(n=18,18 \%)$ and $\operatorname{AdV~}(n=16,16 \%)$. Pertussis was more frequent in

This study was supported by a grant (No. 94-03-88-29448) from Tehran University of Medical Sciences to Dr. Setareh Mamishi.

Mailing Address: Dr. Setareh Mamishi, Department of Pediatric Infectious Disease, Children's Medical Center Hospital, School of Medicine, Tehran University of Medical Sciences, No. 62, Gharib St., Keshavarz Blvd., Tehran, Iran; Phone: +98 0216642 8996; Fax: +98 0216642 8996; e-mail: smamishi@sina.tums.ac.ir 
spring $(8 \%)$ and summer (6\%). In addition, clinical symptoms of pertussis were the same as some viral pathogens, which can lead to misdiagnosis of infection. Therefore, diagnosis of pertussis should be established on the bases of both the clinical symptoms and the laboratory methods.

\section{Introduction}

Bordetella pertussis is a fastidious gram-negative coccobacillus bacterium that causes pertussis (whooping cough), an acute infection of the respiratory tract in humans (Castillo et al., 2015; Pavic-Espinoza et al., 2015). Pertussis is one of the top ten fatal infections in children (Haghighi et al., 2014). Although it is a vaccinepreventable disease, a considerable number of pertussis cases are yet recorded (Nunes et al., 2016). According to the World Health Organization (WHO), approximately 50 million cases of pertussis occur each year, which results in 300000 deaths (World Health Organization, 2016). Infants $<2$ months are more susceptible to pertussis complications since they did not receive the pertussis vaccines (Nunes et al., 2016). The infant mortality rate of pertussis in developing countries is about 4\% (World Health Organization, 2016). The well-known symptoms of pertussis defined as repeated paroxysmal cough, inspiratory whoop, and post-cough vomiting (Shojaei et al., 2014). The atypical milder infection can develop in persons with the prepared immune system either by vaccination or previous infection (Nunes et al., 2016).

Co-infections with respiratory viruses such as respiratory syncytial virus (RSV), rhinovirus/enterovirus, adenovirus (Adv), influenza virus (INF), and human metapneumovirus (hMPV) frequently occur in infants with pertussis infection (Dube et al., 2016; Nicolai et al., 2016). The clinical manifestations of these viral infections are indistinguishable from pertussis (Pavic-Espinoza et al., 2015); therefore, diagnosis of pertussis infection cannot be done only by clinical symptoms and should be confirmed by laboratory tests (del Valle-Mendoza et al., 2015). Real-time PCR is a rapid and sensitive technique that can be employed for fast detection of $B$. pertussis in order to disrupt its transmission and initiate appropriate therapy (Nikbin et al., 2013).

In this study, we aimed to recognize the identity and frequency of etiologic agents of the pertussis-like syndrome in children $<2$ years of age who admitted to Children's Medical Center (CMC) Hospital.

\section{Material and Methods}

Study design and population

This was a cross-sectional hospital-based study designed to recognize the identity and frequency of etiologic agents of the pertussis-like syndrome in all children $<2$ years of age who were suspected to pertussis and admitted to CMC Hospital between August 2014 and August 2015. CMC Hospital is a tertiary referral children's hospital that is located in Tehran, the capital city of Iran. 
Detailed demographic and clinical data such as age, gender, pertussis vaccination status, previous antibiotic consumption, history of pertussis in the family, and clinical symptoms were obtained from questionnaires that were answered by patient's parents and from medical records. Children who met some of the WHO clinical case criteria for pertussis - i.e. cases with a cough more than two weeks with at least one of the following symptoms: paroxysm of a cough (many, rapid coughs followed by a high-pitched "whoop" sound); or inspiratory whoop (a forceful inspiration of air through a narrow glottis, usually developing after a paroxysmal cough); or vomiting after a cough; or apnea (long cessation of breathing) (with or without cyanosis (blue/purplish skin coloration due to lack of oxygen)) were included into the study (Shojaei et al., 2014; World Health Organization, 2016).

\section{Ethical statement}

This study was permitted by the Ethical Committee of the Research Center for Pediatric Infectious Diseases in Tehran University of Medical Sciences. Written informed consent was obtained from parents of all children.

\section{Sample collection}

For each patient, one Dacron swab was obtained from the posterior part of the nasopharynx and inoculated onto the $1 \mathrm{ml}$ of sterile normal saline $(0.9 \% \mathrm{~W} / \mathrm{V} \mathrm{NaCl}$ solution). All specimens were transported at room temperature within 1-2 hours to the microbiology laboratory of the Research Center for Pediatric Infectious Diseases at CMC Hospital and stored at $-70^{\circ} \mathrm{C}$.

\section{TaqMan real-time $P C R$}

A TaqMan real-time PCR assay was applied to detect the identity of pathogens. For each specimen, both RNA and DNA were extracted from the elution liquid of swabs, using the MagjET viral DNA and RNA purification kit (Thermo Scientific ${ }^{\mathrm{TM}}$, USA) and RTP bacteria DNA mini kit (STRATEC Biomedical AG, Germany) according to the manufacturer's protocols. In the case of RNA samples, a randomprimed reverse transcription reaction was performed using the RevertAid reverse transcriptase kit (Thermo scientific ${ }^{T M}$, USA) and cDNA was synthesized according to the manufacturer's instructions. The real-time PCR was performed based on the protocols of Adv, RSV, INF-A, hMPV and B. pertussis real-time PCR kits (Liferriver biotech, USA). An ABI step one real-time PCR system (Applied Biosystems, Foster City, California, USA) was used for this purpose.

\section{Results}

Study population

During the study period, a total of 100 children $<2$ years of age (mean age 5.5 months) presenting some of the symptoms of pertussis such as a paroxysmal 
Table 1 - General and clinical characteristics of the patients

\begin{tabular}{llc}
\hline Characteristics & & Frequency (\%) \\
\hline Gender & Male & 56 \\
& Female & 44 \\
\hline Age & $0-2$ month & 34 \\
& $2-4$ month & 30 \\
& $4-6$ month & 12 \\
& $6-18$ month & 20 \\
& $\geq 18$ month & 4 \\
\hline Pertussis vaccination & Yes & 66 \\
\hline Contact with pertussis patients & Yes & 68 \\
\hline History of antibiotic consumption & Yes & 80 \\
\hline Clinical symptoms & Paroxysmal cough & 100 \\
& Cyanosis & 44 \\
& Vomits & 52 \\
& Apnea & 30 \\
\hline
\end{tabular}

cough, or post-tussive vomiting, or cyanosis, or apnea were included in the study. Demographics and clinical characteristics of the patients are summarized in Table 1. Fifty-six percent of cases were male, and $44 \%$ were female. Thirty-four percent of the patients were in the age group of 0 to 2 months and did not receive any doses of pertussis vaccine. The other remaining $66 \%$ of patients received doses of DTwP vaccine according to their age groups. Eighty percent of the children had a history of antibiotic consumption. A paroxysmal cough was the main complaint of patients and was observed in all cases. The duration of coughing varied from 6 to 30 days (mean duration $8.4 \pm 3.9$ days). The frequency of apnea was lower than other symptoms $(30 \%)$.

\section{TaqMan real-time $P C R$}

From the 100 nasopharyngeal swabs tested by real-time PCR, $75 \%$ were positive for the presence of bacterial or viral nucleic acids. Among the detected pathogens, RSV showed the highest frequency $(20 \%)$, followed by B. pertussis $(18 \%)$, AdV $(16 \%)$, INF-A (11\%), and hMPV (10\%). A dual infection was observed in 8 patients $(11 \%)$, with combinations of RSV/INF-A ( $n=3,4 \%)$, and $A d V / B$. pertussis $(n=3,4 \%)$ being more frequent, followed by RSV/B. pertussis $(n=1,1 \%)$ and $\operatorname{AdV/RSV~}(n=1,1 \%)$. One patient $(1 \%)$ had a triple infection (AdV/RSV/B. pertussis). No simultaneous infection with hMPV was detected. In $25 \%$ of cases, no etiologic agent identified. Most cases of pertussis were observed during spring $(n=8,8 \%)$, followed by summer $(n=6,6 \%)$, autumn $(n=1,1 \%)$, and winter $(n=3,3 \%)$. 


\section{Discussion}

Although a paroxysmal cough is regarded as the most important classical symptom of pertussis and is used for pertussis clinical case definition by WHO and center for disease control and prevention (van den Brink et al., 2014), results of this study demonstrated that it is not a specific symptom for pertussis and of the 100 suspected cases of pertussis, only $18 \%$ were correctly predicted by this criterion. In addition, other symptoms were non-specific to pertussis and they were also observed in some children with viral respiratory infections. In agreement with this study, other reports have emphasized on the lack of specificity of these classical symptoms (Shojaei et al., 2014; van den Brink et al., 2014; Vittucci et al., 2016).

If pertussis diagnosis is based only on the clinical manifestations, atypical and mild infections would be unrecognized (van den Brink et al., 2014) especially in vaccinated children that show mild or less severe infection (van den Brink et al., 2014); or other respiratory infections can be confused with pertussis (Shojaei et al., 2014). This can lead to the consecutive spreading of the pathogen in the population and increases the danger of hospital outbreaks (van den Brink et al., 2014;Vittucci et al., 2016). The identification of pertussis cases should not be based only on the clinical symptoms (Cherry et al., 2012; van den Brink et al., 2014), and laboratory techniques such as real-time-PCR should be used as complementary diagnostic methods.

In this study, B. pertussis was detected only in $18 \%$ of suspected cases. In the study conducted by Hajia et al. (2012) from Tehran, Iran, 12 out of 138 children $<6$ months (9\%) were positive for B. pertussis, which was lower compared to the current result. Castillo et al. (2015) from Peru reported an incidence of pertussis as high as $40 \%$ in patients younger than one year admitted to five hospitals in Lima, Peru, which was higher than the rate of this study. Discrepancies observed among the pertussis frequency could be due to the different studied age ranges, different utilized diagnostic methods that have diverse sensitivity and specificity, and different vaccination coverage in various areas.

As noted, $80 \%$ of patients had used antibiotics before sampling that can cause false-negative results. However, since in the current study identification of the causative agents of the pertussis-like syndrome was performed by real-time PCR method, application of antibiotics could not affect the results. The rapid detection of etiologic agents of the pertussis-like syndrome plays an important role in stopping irrational antibiotic usage and adopting the correct therapeutic approaches.

Whooping cough vaccination program in Iran consists of three doses of DTwP vaccine (diphtheria and tetanus toxoid in combination with whole-cell pertussis) at the second, $4^{\text {th }}$ and $6^{\text {th }}$ months of life. Booster doses are given at 18 months and at 4 to 6 years of age (Sedaghat et al., 2014). In the current study, $89 \%(n=16)$ of pertussis cases were observed in the age group 0 to 6 -month that were not 
fully immunized and received only the three initial doses of DTwP vaccine. Some studies demonstrated that based on the efficiency of vaccine used, the infection can develop soon after the vaccination (van den Brink et al., 2014). Due to the waning efficacy of pertussis vaccine, adults play a significant role in the transmission of disease to the young infants (Sedighi and Sadrosadat, 2015). Therefore, some strategies are suggested for protection of unimmunized young infants, including revaccination of juveniles and adults with DTaP vaccine (acellular pertussis vaccine) to prevent household spread of infection, immunization of pregnant women during the third trimester of gestation that leads to the transfer of maternal anti-pertussis antibodies to the fetus to grant protection of newborns from pertussis prior their vaccination (Sedighi and Sadrosadat, 2015; Vittucci et al., 2016). Since whole-cell pertussis vaccine is not suggested for adults; and there is no access to acellular vaccine in Iran, it is recommended to introduce this type of vaccine into the Iranian national vaccination schedule (Sedighi and Sadrosadat, 2015).

In this research, RSV was the most prominent respiratory pathogen and was identified in $20 \%$ of the cases. In the previous study conducted at CMC Hospital (Pourakbari et al., 2014), RSV was the most prevalent viral pathogen (17.2\%) isolated from nasopharyngeal aspirates of children $<5$ years. According to the results, it can be stated that RSV is the principal cause of acute respiratory infection in children (Parsania et al., 2016); and further studies are required to determine the prevalence and genotype distribution of RSV in Iran (Salimi et al., 2016).

In this study, Adv and INF-A were detected in $16 \%$ and $11 \%$ of the patients, respectively. In the previous study carried out in CMC Hospital (Pourakbari et al., 2014), Adv and INF-A showed the same frequencies (3.4\%) and was lower than the rates of this study. In the study conducted by Naghipour et al. (2007) from Gilan, Iran, all the children $<5$ years of age attending the out-patient clinics and those admitted to the hospital wards were tested; and Adv and INF-A was identified in $14 \%$ and $4 \%$ of cases, respectively. In the study performed by Moattari et al. (2015) from Shiraz, Iran, nasopharyngeal swabs of 435 hospitalized children under 5 years of age were evaluated and $22 \%$ of patients were positive for Adv, which was higher than the rates of this study.

In this report, $10 \%$ of patients were positive for hMPV. Sanaei Dashti et al. (2016) from Shiraz, Iran, investigated 200 children under 12 years of age with upper respiratory tract complaints referred to Infectious Clinic of Mofid Children Hospital and reported a similar frequency for hMPV. However, in the studies conducted by Sultani et al. (2015) from Tehran, Iran (0\%), and Vittucci et al. (2016) from Italy (3.4\%) lower frequencies were reported. Furthermore, higher frequency for hMPV was reported by Moattari et al. (2015) from Shiraz, Iran (15.7\%). The aforementioned differences in the frequency of Adv, INF-A, and hMPV in different studies could be due to the annual variations in the incidence of infections, the age of studied patients, different diagnostic methods used, the size of understudy population, and climate variations (Parsania et al., 2016). 
In the current study, co-infections with RSV/INF-A, AdV/B. pertussis, RSV/B. pertussis, AdV/RSV, and AdV/RSV/B. pertussis were detected in 8 patients (12\%). Several studies revealed that in respiratory infections often more than one pathogen is detected. For instance, Korppi and Hiltunen (2007) reported the co-infection of RSV/B. pertussis in 7 out of 117 infants $<6$ months of age who had lower respiratory tract infections. In the case of mixed infections, it is unclear which pathogen is the principal contributing agent and what is their association with disease severity. It is also unclear which pathogen is more important for the emergence of disease manifestations (van den Brink et al., 2014). Therefore, to avoid unrecognized cases, pertussis should be considered in all infants with respiratory tract infections and the diagnosis of viral pathogens does not mean that pertussis is dismissed.

In this research, the frequency of pertussis in spring and summer was higher than in the other seasons. This was similar to the reports of Ghorbani et al. (2016) from Iran that examined the nasopharyngeal swabs of all notified pertussis cases from different age groups between the years 2011 and 2013 and most of the confirmed cases of pertussis were identified during the spring and summer seasons. Gonfiantini et al. (2014) from Italy, evaluated the data on notified pertussis cases from 1888 to 2012 and observed similar season distribution to the current study. However, in the study performed by Goktas and Sirin (2016) from Turkey, most cases of pertussis ( $40 \%)$ were identified in winter. Consequently, being aware of the seasons with high rates of pertussis, it can be helpful to employ appropriate programs for active case finding and early treatment of patients in order to control quickly the spread of infection.

\section{Conclusion}

In this study, RSV was the most frequently identified pathogen $(n=20,20 \%)$, followed by pertussis $(n=18,18 \%)$ and $\operatorname{AdV}(n=16,16 \%)$. Pertussis was more frequent at spring (8\%) and summer (6\%). In addition, clinical symptoms of pertussis were the same as that of some viral pathogens, and this can lead to misdiagnosis of infection. Therefore, diagnosis of pertussis should be based on both the clinical symptoms and the laboratory methods. Moreover, effective strategies for induction of immunity in unvaccinated young infants including revaccination of juveniles and adults with DTaP, and immunization of pregnant women during the third trimester of gestation; should be introduced into the public health control programs.

\section{References}

Castillo, M. E., Bada, C., Del Aguila, O., Petrozzi-Helasvuo, V., Casabona-Ore, V., Reyes, I., del Valle-Mendoza, J.

(2015) Detection of Bordetella pertussis using a PCR test in infants younger than one-year-old hospitalized with whooping cough in five Peruvian hospitals. Int. J. Infect. Dis. 41, 36-41. 
Cherry, J. D., Tan, T., Wirsing von König, C. H., Forsyth, K. D., Thisyakorn, U., Greenberg, D., Johnson, D., Marchant, C., Plotkin, S. (2012) Clinical definitions of pertussis: Summary of a global pertussis initiative roundtable meeting, February 2011. Clin. Infect. Dis. 54(12), 1756-1764.

del Valle-Mendoza, J., Casabona-Oré, V., Petrozzi-Helasvuo, V., Cornejo-Tapia, A., Weilg, P., Pons, M. J., Cieza-Mora, E., Bazán-Mayra, J., Cornejo-Pacherres, H., Ruiz, J. (2015) Bordetella pertussis diagnosis in children under five years of age in the Regional Hospital of Cajamarca, Northern Peru. J. Infect. Dev. Ctries. 9(11), 1180-1185.

Dube, F. S., Kaba, M., Robberts, F. L., Tow, L. A., Lubbe, S., Zar, H. J., Nicol, M. P. (2016) Respiratory microbes present in the nasopharynx of children hospitalised with suspected pulmonary tuberculosis in Cape Town, South Africa. BMC Infect. Dis. 16(1), 597.

Ghorbani, G. R., Zahraei, S. M., Moosazadeh, M., Afshari, M., Doosti, F. (2016) Comparing seasonal pattern of laboratory confirmed cases of pertussis with clinically suspected cases. Osong Public Health Res. Perspect. 7(2), 131-137.

Goktas, S., Sirin, M. C. (2016) Prevalence and seasonal distribution of respiratory viruses during the 2014-2015 season in Istanbul. Jundishapur J. Microbiol. 9(9), e39132.

Gonfiantini, M.V., Carloni, E., Gesualdo, F., Pandolfi, E., Rizzuto, E., lannazzo, S., Degli Atti, M. C., Villani, A., Tozzi, A. E. (2014) Epidemiology of pertussis in Italy: disease trends over the last century. Euro Surveill. 19(40), 20921.

Haghighi, F., Shahcheraghi, F., Abbasi, E., Eshraghi, S. S., Zeraati, H., Mousavi, S. A. J., Asgarian-Omran, H., Douraghi, M., Shokri, F. (2014) Genetic profile variation in vaccine strains and clinical isolates of Bordetella pertussis recovered from Iranian patients. Avicenna J. Med. Biotechnol. 6(3), 178.

Hajia, M., Rahbar, M., Fallah, F., Safadel, N. (2012) Detection of Bordetella pertussis in infants suspected to have whooping cough. Open Respir. Med. J. 6, 34-36.

Korppi, M., Hiltunen, J. (2007) Pertussis is common in nonvaccinated infants hospitalized for respiratory syncytial virus infection. Pediatr. Infect. Dis. J. 26(4), 316-318.

Moattari, A., Aleyasin, S., Emami, A., Fyruzi, M., Pirbonyeh, N. (2015) The prevalence of human metapneumovirus and respiratory syncytial virus and coinfection with both in hospitalized children with acute respiratory infection in south of Iran. Arch. Pediatr. Infect. Dis. 3(3), e32974.

Naghipour, M., Cuevas, L. E., Bakhshinejad, T., Mansour-Ghanaei, F., Noursalehi, S., Alavy, A., Dove, W., Hart, C.A. (2007) Contribution of viruses, Chlamydia spp. and Mycoplasma pneumoniae to acute respiratory infections in Iranian children. J. Trop. Pediatr. 53(3), 179-184.

Nicolai, A., Nenna, R., Frassanito, A., Pierangeli, A., Mileto, F., Scagnolari, C., Mancino, E., Di Mattia, G., Petrarca, L., Cangiano, G., Midulla, F. (2016) Respiratory viruses and Bordetella pertussis co-infections: A frequent occurrence in children hospitalized with Bordetella pertussis. Eur. Resp. J. 48(S60), PA1275.

Nikbin, V. S., Shahcheraghi, F., Lotfi, M. N., Zahraei, S. M., Parzadeh, M. (2013) Comparison of culture and real-time PCR for detection of Bordetella pertussis isolated from patients in Iran. Iran. J. Microbiol. 5(3), 209-214.

Nunes, M. C., Downs, S., Jones, S., van Niekerk, N., Cutland, C. L., Madhi, S. A. (2016) Bordetella pertussis infection in South African HIV-infected and HIV-uninfected mother-infant dyads: a longitudinal cohort study. Clin. Infect. Dis. 63, 174-180 (Suppl. 4).

Parsania, M., Poopak, B., Pouriayevali, M. H., Haghighi, S., Amirkhani, A., Nateghian, A. (2016) Detection of human metapneumovirus and respiratory syncytial virus by real-time polymerase chain reaction among hospitalized young children in Iran. Jundishapur J. Microbiol. 9(3), e32974.

Pavic-Espinoza, I., Bendezú-Medina, S., Herrera-Alzamora, A., Weilg, P., Pons, M. J., Aguilar-Luis, M. A., PetrozziHelasvuo, V., del Valle Mendoza, J. (2015) High prevalence of Bordetella pertussis in children under 5 years old hospitalized with acute respiratory infections in Lima, Peru. BMC Infect. Dis. 15(1), 554. 
Pourakbari, B., Mahmoudi, S., Movahedi, Z., Halimi, S., Momeni, S., Hosseinpour-Sadeghi, R., Mamishi, S. (2014) Viral etiology of acute lower respiratory tract infections in hospitalized young children in a children's referral hospital in Iran. Turk. J. Pediatr. 56(4), 354-359.

Salimi, V., Tavakoli-Yaraki, M., Yavarian, J., Bont, L., Mokhtari-Azad, T. (2016) Prevalence of human respiratory syncytial virus circulating in Iran.J. Infect. Public Health 9(2), 125-135.

Sanaei Dashti, A., Emamifar, S. M. H., Hashempour, T., Malekan, M. A. (2016) Human metapneumovirus frequency in Iranian children with respiratory symptoms. Future Virol. 11(3), 181-186.

Sedaghat, M., Lotfi, M. N., Talebi, M., Saifi, M., Pourshafie, M. R. (2014) Status of pertussis in Iran. Jundishapur J. Microbiol. 7(11), e12421.

Sedighi, I., Sadrosadat, T. (2015) Acellular pertussis vaccine: Iran's next step to control the disease? Arch. Pediatr. Infect. Dis. 3(2), e23918.

Shojaei, J., Saffar, M. J., Hashemi, A., Ghorbani, G. R., Rezai, M. S., Shahmohammadi, S. (2014) Clinical and laboratory features of pertussis in hospitalized infants with confirmed versus probable pertussis cases. Ann. Med. Health Sci. Res. 4(6), 910-914.

Sultani, M., Azad, T. M., Eshragian, M., Shadab, A., Naseri, M., Eilami, O., Yavarian, J. (2015) Multiplex SYBR green real-time PCR assay for detection of respiratory viruses. Jundishapur J. Microbiol. 8(8), e19041.

van den Brink, G.,Wishaupt, J. O., Douma, J. C., Hartwig, N. G., Versteegh, F. G. (2014) Bordetella pertussis: An underreported pathogen in pediatric respiratory infections, a prospective cohort study. BMC Infect. Dis. 14(1), 526.

Vittucci, A. C., Vennarucci, V. S., Grandin, A., Russo, C., Lancella, L., Tozzi, A. E., Bartuli, A., Villani, A. (2016) Pertussis in infants: an underestimated disease. BMC Infect. Dis. 16(1), 414.

World Health Organization (2016) WHO-recommended Surveillance Standard of Pertussis. 